\title{
Internal coordinates
}

The grid constructed in the space of 6 dimer coordinates: the " $z y z$ " Euler angles $\beta_{A}$ and $\gamma_{A}$ of monomer $A$, the " $z y z$ " Euler angles $\alpha_{B}, \beta_{B}$, and $\gamma_{B}$ of monomer $B$, and the distance $R$ between the centers of mass of the monomers. The "zyz" Euler angles of a given monomer are defined in a local coordinate frame with the origin in the center of this monomer and the $z$ axes parallel to the vector pointing from the center of $A$ to the center of $B$. Both local

frames are parallel to each other. When all three Euler angles of a monomer are equal to zero, this monomer is located in the $x y$ plane of its local frame with two carbon atoms on the $y$ axis. With dimer coordinates defined in this way, the coordinate $\alpha_{A}$ can be arbitrarily set to zero since the dependence of the potential on this coordinate is only through the difference $\alpha_{B}-\alpha_{A}$. 